\title{
Novel Applications of Focused Ion Beam Technique for Planetary Sample Analyses
}

\author{
Z. Rahman ${ }^{1}$, L. P. Keller ${ }^{2}$, A. N. Nguyen ${ }^{1}$, M. S. Thompson ${ }^{2}$ and Scott R. Messenger ${ }^{2}$ \\ 1. Science Department, Jacobs, NASA-JSC, USA. \\ 2. ARES, NASA-JSC, Houston, USA.
}

We are using innovative FIB techniques to prepare samples of planetary materials for different types of coordinated analyses using ion microprobes, synchrotron beamlines, and specialized transmission electron microscopy (TEM) techniques. In these cases, the FIB sample preparation is the critical step in enabling these specialized analyses. We discuss several examples below utilizing the FEI Quanta3D instrument at the NASA Johnson Space Center.

The trace element content of mineral grains in comet dust provides important clues on their formation and processing in the early solar system. We preformed coordinated analyses of a comet dust particle that had been prepared using ultramicrotomy for TEM analysis. Following the TEM analyses, we extracted a $70 \mathrm{~nm}$ thick section from a region of the carbon (C) film of the TEM grid, for additional analyses. A carbon ring $\sim 2-3 \mu \mathrm{m}$ thick was deposited on top of the $\mathrm{C}$ film using the FIB. The $\mathrm{C}$ film on the outer rim of the ring was milled away using various patterns to uniformly release the stresses on the film, preventing rupture and collapse, and was attached to the micromanipulator needle (Fig. 1a). We then isolated the ring completely (Fig. 1b) and transferred the section to a silicon sample holder (Fig. 1c) for analysis using the HXN (hard X-ray nanoprobe) beamline at NSLSII at Brookhaven National Lab [1, $2]$.

Rare sub- $\mu \mathrm{m}$ presolar grains that originate in evolved stars and supernovae, occur in primitive astromaterials and are identified by their exotic isotopic compositions. Coordinated analyses of these grains using NanoSIMS, TEM, and other techniques on the same grain is enabled by innovative FIB sample preparation [3]. In order to obtain accurate isotopic analyses of $\mathrm{Mg}$ and $\mathrm{Fe}$, the FIB was used to remove surrounding contaminant grains. We precisely deposited a protective cap of Pt on top of the grain to preserve the grain of interest and then milled away about $5 \mu \mathrm{m}$ diameter of the surrounding material (Fig. 2a). Following the isotopic analyses, the spindle was extracted and thinned to electron transparency for TEM microstructural analyses (Fig. 2b, c).

We extracted a FIB thin section from Apollo 17 lunar rock 76015. To avoid ion-beam damage, e-beam deposition was used to deposit the first $500 \mathrm{~nm}$ of the $\mathrm{C}$ strap, followed by ion beam-assisted deposition of $\sim 3 \mu \mathrm{m}$ carbon. We performed an ex situ lift-out of the section and placed the section on one of the elements of a microelectromechanical systems (MEMS) - specialized heating substrate (Fig. 3a) and attached the section to the substrate by depositing small $\mathrm{C}$ straps with the FIB (Fig. 3b, c). The heating chips utilize silicon nitride windows to support the samples and provide uniform heating while enabling TEM imaging. The heating chip was loaded into a Hitachi "Blaze" heating holder and analyzed using a Hitachi HF5000 at the University of Arizona [4].

References:

[1] M. Schoonen, M. et al, Lunar \& Planetary Science Conf. (2018) \#2951.

[2] G. J. Flynn et al, Meteoritics Planet. Sci. (2016) \#6205. 
[3] A. N. Nguyen et al, Astrophysical J. 818 (2016), p. 1.

[4] L. P. Keller et al, Lunar \& Planetary Science Conf. (2018) \#2594.
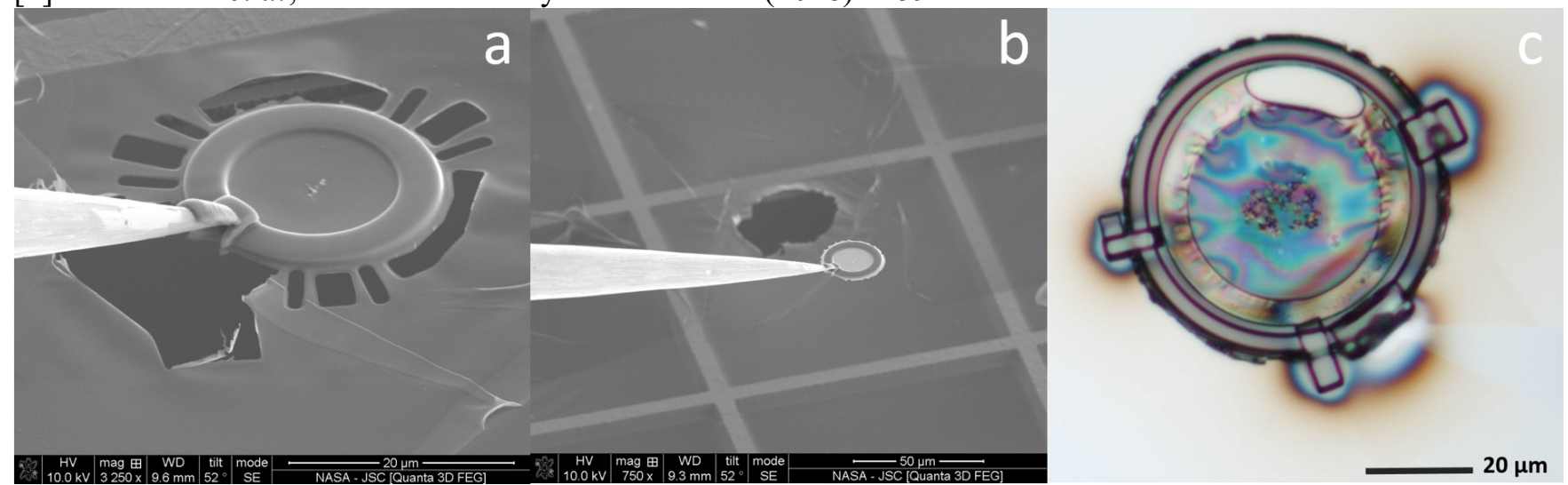

Figure 1. a) thin section with deposited C-ring attached to the needle. b) assembly lifted from the C film TEM grid. c) reflected light image of the extracted section attached to a Si substrate for analysis.

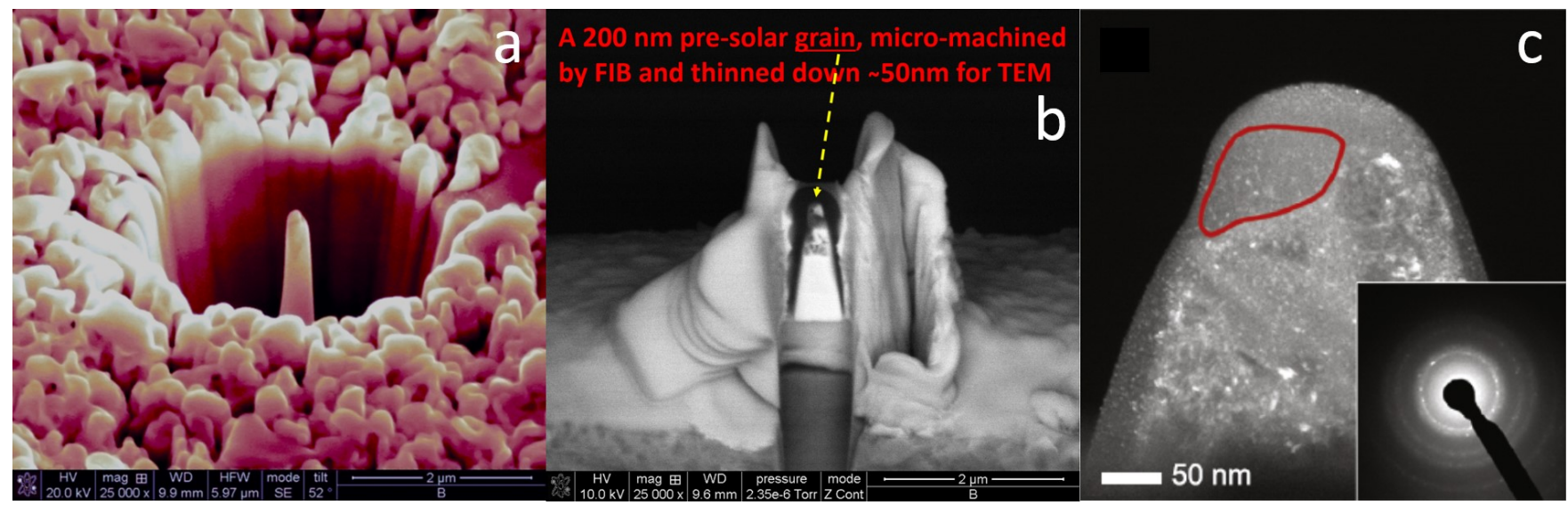

Figure 2. a) a sub- $\mu \mathrm{m}$ presolar grain is isolated from neighboring meteorite matrix grains by FIB milling for subsequent NanoSIMS isotopic analysis. b) an electron transparent section of the grain was then produced by FIB for mineralogical analysis by TEM. c) darkfield TEM image and diffraction pattern from the presolar grain [3].

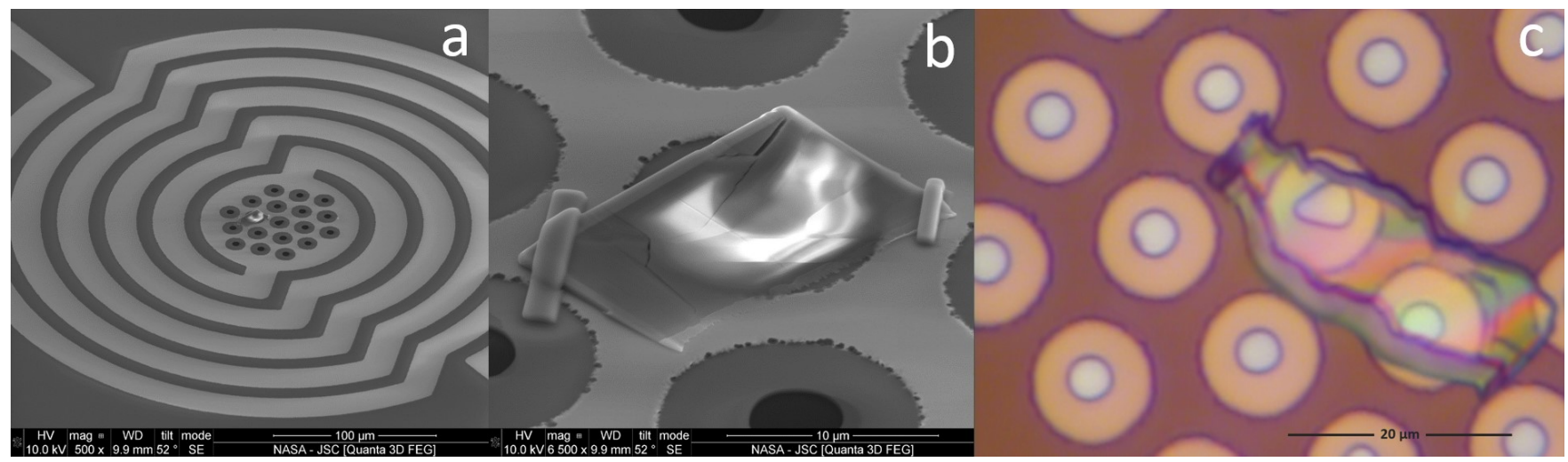

Figure 3. a) SEM image of the heating chip. b) SEM image of the FIB section attached to the heating chip with C straps. c) reflected light image showing the FIB section placed on the heating chip. 